\title{
Bericht
}

Monika Henkel

\section{Argumentstrukturen zwischen Valenz und Konstruktionen}

\author{
Empirie - Theorie - Anwendung \\ Tagung an der Universität Santiago de Compostela \\ 26.-28. September 2012
}

\begin{abstract}
Monika Henkel: Universität Kassel, Fachbereich 2 - Germanistik, Kurt-Wolters-Str. 5, D-34125 Kassel, E-Mail: monika-henkel@uni-kassel.de
\end{abstract}

An der Universität Santiago de Compostela fand vom 26.09.-28.09.2012 die internationale Fachtagung der germanistischen Linguistik Argumentstrukturen zwischen Valenz und Konstruktionen (Empirie - Theorie - Anwendung) statt, die im Rahmen der Forschungskooperation der Universität Santiago de Compostela und des Instituts für Deutsche Sprache in Mannheim zustande kam und deren Organisation Meike Meliss und Stefan Engelberg zu verdanken war. Das Ziel der Fachtagung war, grammatische Phänomene unter Gesichtspunkten der Valenzund Konstruktionsgrammatik zu beleuchten und zu prüfen, in welchen theoretischen Problemstellungen und Anwendungsbereichen sich Valenz- und Konstruktionsgrammatik gegenseitig anregen und ergänzen können.

Den Auftakt bildete Anatol Stefanowitsch mit einem Vortrag über Argumentstrukturkonstruktionen als (emergente) Generalisierungen über Valenzstrukturen. Die Frage nach der Verortung der Argumentstruktur werde von Valenz- und Konstruktionsgrammatik unterschiedlich beantwortet. Während die Valenzgrammatik Argumentstrukturen als Eigenschaften einzelner Lexeme sehe, hätten in der Konstruktionsgrammatik Argumentstrukturen, unabhängig von einzelnen Lexemen, einen eigenen Status. Beide Konzepte öffneten unterschiedliche Perspektiven auf Sprache. Stefanowitsch plädierte für ein gebrauchsgestütztes kognitiv-grammatisches Modell, das beide Perspektiven im Blick habe.

Im darauf folgenden Vortrag Die Wechselwirkung von Valenz und Konstruktion befasste sich Klaus Welke mit Gemeinsamkeiten und Unterschieden zwischen Valenz- und Konstruktionsgrammatik. Sowohl die Konstruktionsgrammatik als auch die Valenzgrammatik gingen vom Kopfprinzip aus. Die Unterschiede sieht 
Welke, ebenso wie Stefanowitsch, in der Perspektivierung. Während die Valenzgrammatik von dem Wort als Grundeinheit ausgehe, definiere die Konstruktionsgrammatik die Konstruktion als Grundeinheit. In der Frage, ob der Kopf die Konstruktion oder die Konstruktion den Kopf bestimme, nehmen nach Welke beide Grammatiktheorien unterschiedliche Positionen ein. Welke plädierte für eine Fusionierung von Valenz- und Konstruktionsgrammatik auf Basis der Konstruktionsgrammatik und begründete seinen Vorschlag damit, dass die Projektion der Konstruktion folge, d.h. die Konstruktion sei primär und die Projektion sekundär. Deutlich werde dies unter anderem bei kopflosen Konstruktionen (z. B. Jedem das Seine), und Konstruktionen mit „überschüssigen“ Argumenten (z. B. freier Dativ), d. h. mit Argumenten, die als nicht „vorhersehbar“ gelten.

Einen Beitrag zur Valenz-Empirie präsentierte Raúl Sánchez Prieto mit den Ergebnissen seiner Untersuchung zu Deutsche, spanische und niederländische Valenz- und Argumentstrukturen im Web 2.0: Ein Pilotprojekt. Sánchez Prieto untersuchte in dieser kontrastiven, textsortenspezifischen Studie Valenz- und Argumentstrukturen von Userkommentaren in Hotelbewertungsportalen und beschäftigte sich mit kulturell-sprachlichen Unterschieden in Kundenargumenten. Seine Belege zeigten, dass es in dem kontrastiven Bereich sowohl Übereinstimmungen als auch Abweichungen in den grammatisch-semantischen Strukturen gebe. So wies Sánchez Prieto z. B. nach, dass deutsche Userkommentare Hotelempfehlungen expliziter ausdrücken als spanische oder niederländische Userkommentare.

Der Vergleich von Valenzrealisierungen von Bewegungsverben mit der Analyse von grammatischen Konstruktionen von Bewegungsereignissen stand im Mittelpunkt des Beitrags von Marta Fernández Villanueva: Bewegungsverben: regelhafte und idiosynkratische Aspekte. Fernández Villanueva untersuchte sowohl die Rolle der Valenz als auch die Integration des situationellen Wissens im Hinblick auf die nicht-prototypische Verwendung von Bewegungsverben in dem multimodalen Korpus VARKOM. Die nicht-prototypische Verwendung von Bewegungsverben entstehe in der Interaktion und hänge stark von der Perspektivierung ab. Daher habe die Darstellung von nicht-prototypischen Verwendungen von Bewegungsverben mit valenziellen und konstruktionsgrammatischen Beschreibungsansätzen bzw. im Rahmen der kompositionellen oder der kognitiven Semantik sowohl Stärken als auch Schwächen. Fernández Villanueva versteht die nicht-prototypische Verwendung von Bewegungsverben mit ihren fokussierenden und aufmerksamkeits-steuernden Elementen stärker als Perspektivierungsproblem denn als Grammatikproblem.

Mit einem anwendungsorientierten Beitrag von Meike Meliss Argumentstrukturen, Valenz und Konstruktionen im Umfeld von Verben der Wahrnehmung im Deutschen und Spanischen. Eine kontrastive Studie im Rahmen von DICONALE 
endete der erste Tagungstag. Bilaterale Wörterbücher erforderten eine stärker anwenderorientiere Perspektive der Lexikographie. Dies machte Meliss am Beispiel des Wahrnehmungsverbes schnuppern deutlich, über dessen Verwendungsweise als Bewegungsverb (schnuppern + Direktiva) Wörterbücher keine Auskunft geben. Bei diesen Konstruktionen gebe es aus fremdsprachlicher Sicht Probleme für den Produktions- und Rezeptionsprozess, die sich u. a. mit der übertragenen Bedeutung erklären ließen. Im Rahmen des Forschungsprojektes DICONALE entstehe ein konzeptionell orientiertes Wörterbuch der deutschen und spanischen Sprache, welches z. B. für Konstruktionen wie schnuppern + Direktiva in verschiedenen Beschreibungsmodulen Informationen zur Argumentstruktur, zur semantischen Füllung, zu Aktanten und zur morphosyntaktischen Valenz gebe. Aus einer anwenderorientierten Sicht müsse das Angebot an lexikographischen Möglichkeiten verstärkt werden.

Den zweiten Tagungstag eröffnete Vilmos Âgel mit seinem Vortrag +/- Konstruktion (Valenz) = +/- Valenzdynamik. Im ersten Teil des Vortrags befasste sich Ágel mit Statik und Dynamik von Satzgliedern, um im zweiten Teil des Vortrags zu zeigen, wie sich das Modell Statik/Dynamik auf die freien Dative (Dativ commodi/incommodi und Pertinenzdativ) auswirkt. Zunächst stellte er die Grundidee der Unterscheidung von statischen und dynamischen Sätzen dar. Während bei statischen Sätzen die Valenz 1:1 umgesetzt werde, finde bei dynamischen Sätzen eine Umstrukturierung statt, die Valenz werde nicht 1:1 realisiert. Die Beschreibung mithilfe der Funktions-Argument-Wert-Formel, die auch im Vortragstitel erscheint, differenziert nach Ágel zwischen grammatischen Formen (Argumente), die bestimmte Funktionen in Sätzen übernehmen, und deren Werten, die Satzgliedern entsprechen. Die statische Valenz vererbe sich auf den dynamischen Satz. Konstruktion und Valenz verhielten sich zueinander wie Dynamik und Statik. Als Beispiel für Valenzdynamik modellierte Ágel die freien Dative und die Dativalternationen (Transfer- und Benefaktiv-Alternation) als Valenzphänomene der dritten Art. Im Gegensatz zu Welke plädierte Ágel für eine Fusionierung von Konstruktions- und Valenzgrammatik auf Basis der Valenzgrammatik.

Klaus Fischer ging in seinem Beitrag Zwischen Valenz und Konstruktion: Argumente im Fokus auf Informationsstrukturen mit kontrastiven Fokuskonstruktionen ein. Über eine Spaltsatzkonstruktion könne ein kontrastiver Fokus gesetzt werden. Fischer versteht diese Spaltsatzkonstruktionen als zweiwertige Prädikatstruktur mit einem Hintergrund, der präsupponiert wird und einer Spezifizierung in Form einer kontrastiven Fokuskonstruktion. (Beispiel: Es ist der Spiegel, der im Sommer 2003 das Geheimnis lüftete). Diese Fokussierung könne auch durch eine Fokuspartikel in Form eines Hervorhebungsakzentes realisiert werden. Im Rahmen eines Sprachvergleichs untersuchte Fischer, ob englische Spaltsätze kon- 
trastiven deutschen Fokuskonstruktionen entsprechen. Als Beispiel nannte Fischer die Untersuchung der deutschen und englischen Ausgabe von Alex Capus „Eine Frage der Zeit“. In der englischen Übersetzung ließen sich erheblich mehr Spaltsätze belegen als im deutschen Original.

Ansätze zur Beschreibung des Kombinationspotenzials von Fortbewegungsverben. Ein kontrastiver Versuch war der Gegenstandsbereich des Vortrags von Juan Cuartero Otal. Cuartero Otal prüfte die Hypothese der Eignungsfähigkeit eines lexikalisch- und valenzbasierten Modells für spanische Verben und eines konstruktionsgrammatischen Modells für deutsche Verben und beschäftigte sich mit Beschreibungsmodellen für deutsche und spanische Fortbewegungsverben. Einer gut untersuchten Gruppe der Fortbewegungsverben stellte er eine Gruppe von Verben der Eigenfortbewegung entgegen, die er Bummel-Verben nannte (Beispiele: flanieren, bummeln) und die in ihrer Kombinatorik Besonderheiten aufwiesen. Cuartero Otal schlug für die Beschreibung von Bummel-Verben eine detaillierte Auflistung von lizensierten Komplementen für jedes einzelne Verb vor.

Arne Zeschel erläuterte in seinem Beitrag Probleme der Randbereichsunschärfe in konstruktionsbasierten Analysen Abgrenzungsprobleme von Konstruktionen. Am Beispiel einiger Verben (z. B. schwirren, strotzen, zittern) belegte Zeschel, dass diese Verben auch Satzglieder möglich machen, die in der Valenzpotenz dieser Verben nicht verankert sind. Eine konstruktionsbasierte Analyse habe u. a. den Vorteil, dass Vorkommensmuster in ihrer unmittelbaren systemischen Umgebung kontextualisiert würden und Bedeutungseigenschaften beschrieben werden könnten, die nicht von den lexikalischen Köpfen motiviert seien. Randbereichsunschärfen ergeben sich nach Zeschel z. B. aus der semantischen Gradienz oder auch aus der Unzuverlässigkeit vermeintlich maßgeblicher Tests.

Mit Vererbung, Hierarchie, Variation und konstruktionelle Polysemie: zu netzwerkartigen Verknüpfungsbeziehungen mit Hinblick auf die Konstruktions- und Valenzgrammatik beschäftigte sich der Beitrag von María J. Domínguez Vázquez. Im Fokus stand die Prüfung der Praxistauglichkeit von Begriffen der Konstruktionsgrammatik. An einem Beispiel von konstruktioneller Polysemie mich friert/ich friere befasste sich Domínguez Vázquez mit der Grenze zwischen Konstruktionsvererbung und Konstruktionsveränderung. Im Hinblick auf das Vererbungskonzept müsse die Reichweite von Konstruktionen geklärt werden.

Als themenübergreifender Vortrag war der Beitrag Deutsch lernen im Internet: Blended Learning. Erfahrungsbericht der UNED und Zukunftsperspektiven von Germán Ruipérez \& J. Carlos García Cabrero angelegt. Blended Learning stehe für einen computergestützten Fremdsprachenunterricht an der Fernuniversität, der die Möglichkeiten des Internets kombiniere mit freiwilligen Präsenzphasen, in denen die Mündlichkeit im Vordergrund stehe. Dabei biete das Internet die Möglichkeit verschiedene Lernplattformen zu nutzen und $\mathrm{zu}$ kom- 
binieren. Wichtig für die Zukunft erscheint Ruipérez auch für mündliche Sprachfertigkeiten web-basierte Werkzeuge stärker zu nutzten.

Grenzen der rein valenzgrammatischen bzw. rein konstruktionsgrammatischen Beschreibungen von Sprachstrukturen lassen sich nach Clemens Knobloch an Beispielen der „Possession“ zeigen. Diese Position führte er mit seinem Beitrag Genitivkonstruktionen und Possessorrealisierungen im Deutschen näher aus und zeigte, dass für die Beschreibung von „Possessions-Konstruktionen“ alle sprachlichen Systemebenen herangezogen werden müssen. Possessionskonstruktionen seien genuine Argumentstrukturen und possessive Beziehungen könnten durch unterschiedliche Konstruktionen realisiert werden. Die attributive Genitivkonstruktion habe dabei eine zentrale Bedeutung. Nach Knobloch können mit Genitivkonstruktionen ganz unterschiedliche Beziehungen zwischen Bezugswort und Spezifizierung ausgedrückt werden. Neben inhärenten Argumentstrukturen gehörten dazu auch grammatisch-semantische Verhältnisse, die sowohl als unmarkiert (das Laster der Trunksucht) als auch - im Sinne von Inhärenz - als markiert (ein Mann der Tat) gelten. Die Vielfalt der sachlichen Beziehungen zeige sich auch in Konstruktionen, deren Bezugswort sich schwer abgrenzen lasse (Der Kirschbaum meines Nachbarn). Aus kategorialer Sicht könne mit der Genitivkonstruktion eine Nominalgruppe näher bestimmt werden und jedes lexikalische Nomen könne Bezugswort $\mathrm{zu}$ einem Genitivattribut sein (Ausnahme: Eigennamen und pronominale Ausdrücke). Possessive Lesarten ließen sich aber auch durch den Kontext aushebeln, wie das Beispiel Caesars Eroberung Roms zeige.

Ludwig M. Eichinger beendete den zweiten Tagungstag mit seinem Vortrag Kookkurenz und Dependenz. Die Dependenzgrammatik biete zunächst die Möglichkeit einer einfachen Darstellung von Abhängigkeiten. In Valenzwörterbüchern, die sich auf generalisierte Vorkommensbedingungen konzentrierten, fänden sich Gebrauchspräferenzen in Form von auffälligen Kookkurrenzen, die sich bei der Untersuchung großer Korpora zeigten, häufig nicht wieder. Neben einer Grundvalenz gebe es Abweichungen von dieser Grundvalenz. Als Beispiel nannte Eichinger das Verb geben. Das VALBU (elektronisches Wörterbuch deutscher Verben) liste für geben 18 Lesarten auf. Korporauntersuchungen belegten, dass geben, obwohl dreiwertig, selten dreiwertig verwendet werde. Verwendungspräferenzen zeigten nicht in Valenzwörterbüchern enthaltene lexikalische Differenzierungen von Verben, deren Bedeutung sich erst im Kontext erschließe.

Der dritte Tagungstag wurde von Irene Rapp mit ihrem Vortrag zu Alternationen bei der Realisierung von Objektsätzen eröffnet. Die Semantik des Matrixverbes bestimme die Präferenz für die Realisierung von Objektsätzen als dass-Satz, in Form eines $z u$-Infinitivs oder als V2-Satz. Rapp unterscheidet zwei Parameter: die Subjektausrichtung und die Sprecherausrichtung. Die Subjektausrichtung lasse sich durch assertive Verben (Deklarativsatz) oder durch volitiv-prospektive Verben 
(Imperativsatz) realisieren. Für die Sprecherausrichtung kämen nur assertive Verben in Frage. Dabei könne zwischen der Realisierung durch faktive und nichtfaktive Verben im Matrixsatz differenziert werden. Nach Rapp erlauben nur die nichtfaktiv assertiven Verben alle drei Möglichkeiten der Komplementrealisierung. Faktiv assertive und volitive Verben ermöglichten keine Komplementrealisierung mit V2 Satz. Als unmarkierte Komplementrealisierung gelten der dass-Satz für assertive Verben und der $z u$-Infinitiv für volitive Verben. Über den Satzmodus könnten Ausnahmen bewirkt werden. Die Begründung für diese Präferenzen sieht Rapp darin, dass volitive Verben im Gegensatz zu assertiven Verben meist eine obligatorische Kontrolle ausübten. Ob die präferierte Komplementergänzung verbspezifisch bzw. klassenspezifisch sei, müsse geprüft werden. Zusammenfassend könne gesagt werden, dass die unmarkierte Komplementselektion semantisch bestimmt werde und durch die Form des Komplements zwischen verschiedenen Lesarten unterschieden werden könne.

Anke Holler warf in ihrem Beitrag Argumentstrukturelle Eigenschaften nichtnativer Verben im Deutschen einen Blick auf die syntaktische und semantische Integration nicht-nativer Verben. Um den Integrationsgrad $\mathrm{zu}$ prüfen, verglich Holler die Argumentstrukturen nativer und nicht-nativer Verben und stellte fest, dass sich bei nicht-nativen Verben keine Dativbildung dokumentieren lasse. Holler führte dazu an, dass sich auch bei nativen Verben eine Verdrängung der Dativobjekte durch Präpositionalobjekte beobachten lasse. Weniger Abweichungen zwischen nativen und nicht-nativen Verben ließen sich bei Argumentstrukturen im Nominativ oder Akkusativ belegen. Die semantischen Rollen des Rezipienten und Benefaktivs zeigten sich bei nicht-nativen Verben unterrepräsentiert. Einen Zusammenhang dieser Ergebnisse mit dem geringen Datenmaterial schloss Holler nicht aus. Die empirische Pilotstudie lasse den Schluss zu, dass nichtnative Verben hinsichtlich ihrer Argumentstruktur als syntaktisch und semantisch integriert gelten können.

Mit Alternanzen wie (a) „er baut eine Hundehütte aus dem Holz“ oder (b) „er verbaut das Holz zu einer Hundehütte“ beschäftigte sich Edeltraud Winkler in ihrem Vortrag Material-Produkt-Alternanz im Deutschen. Material-Produkt-Argumente könnten entweder als direktes Objekt oder als Präpositionalphrase mit verschiedenen Präpositionen realisiert werden. Winkler erläuterte die Erwartbarkeit und Häufigkeit von Verben für diese Material-Produkt-Alternanz. Ihre empirische Untersuchung zeige eine Häufung von wenigen Verben. In Verbindungen wie in Beispielbeleg (b), d. h. das Material nimmt die Stelle des direkten Objektes ein, träten häufig, aber nicht immer, Präfixverben auf. Während sich für das Produkt in den Präpositionalphrasen eine gewisse Stabilität der Präposition $z u$ dokumentieren lasse, könne für das Material eine größere Vielfalt belegt werden. Neben der Präposition aus könne auch mit oder von nachgewiesen werden. 
Überlegungen zum Phänomen der Phrasenkonstruktionen im Deutschen und Spanischen im Rahmen des phraseologsichen Forschungsprojekts FRASESPAL standen im Fokus der Präsentation von Carmen Mellado Blanco. Nach Mellado Blanco eignet sich die Konstruktionsgrammatik besser zum Beschreiben von Phraseologismen als die Valenzgrammatik, die vom Lexem ausgehe. Phraseologismen müssten zunächst von Idiomen abgegrenzt werden. Während Idiome feste Einheiten darstellten und nicht als Grundlage zur Musterbildung dienten, würden Phraseologismen als Muster für weitere Konstruktionen gelten. In einem Korpus aus Internetbelegen untersuchte sie die Phrasemkonstruktion „X versteht von Y (ungefähr) so viel wie ich von Z“ aus strukturell-semantischer Sicht im Hinblick auf interlinguale Übereinstimmungen. Für den Slot $Z$ ließen sich sowohl metaphorische als auch adhoc-Bildungen als Füllung belegen. Im Rahmen der Konstruktionsgrammatik empfiehlt Mellado Blanco weitere kontrastive Studien zu Phrasenkonstruktionen.

Mario Franco Barros stellte seine Untersuchung Topik-Ellipsen und andere Nähemerkmale in der Sprache moderner Medien im Rahmen der Valenz(theorie) vor, die er unter Anwendung des Nähe-Distanz-Modells von Ágel/Hennig an einem Korpus von Emails, Weblogs und Twitter durchführte. Ellipsen stellten im Rahmen seiner Näheanalysen einen entscheidenden Aspekt dar. Die verschiedenen Ellipsen-Definitionen ließen sowohl eine pragmatische als auch eine syntaktische Perspektive zu. Franco Barros diskutierte den bestehenden Konflikt zwischen der Valenz des Verbes und der Angemessenheit der Situation, die im Kontext der pragmatischen Topikellipsen die Realisierungen obligatorischer Ergänzungen nicht erfordern.

In dem Beitrag Auslegungsformeln in der exegetischen Literatur gestern und heute: Valenzwechsel oder Konstruktionspräferenzen? erläuterte Mireia Calvet Creizet die Gründe für die Ersetzung der Präposition dabei durch daran bei Verben der Aufmerksamkeitssteuerung (merken, (er-)kennen) in revidierten Fassungen der Lutherbibel. Neben der Vermutung, dass es sich um einen Wandel des Valenzträgers handele, könne die Begründung auch in einen Wandel der Vertextungstechniken von Auslegungsformeln gesucht werden. Die These, dass vorlutherische Übersetzungen der Bibel als „korrektere“ Umschreibungen im Sinne von 1:1 Übersetzungen gewertet werden können, bestätigte Calvet Creizet im Rahmen ihrer Untersuchung in Bezug auf die Präpositionalphrasen, nicht jedoch in Bezug auf die Verben. Über Kontexte ließe sich ein Bedeutungswandel induzieren. Bei den Argumentstrukturen seien bedeutungstragende Konstruktionen verloren gegangen, damit einher gehe ein Inhaltsverlust.

Paloma Sánchez Hernández setzte sich mit dem Gebrauch und Frequenz von verschiedenen Verbalstrukturen zum Ausdruck von KOGNITION im Deutschen und Spanischen aus kontrastiver Perspektive auseinander. Sánchez Hernández unter- 
suchte im Subfeld „Lernen“ deutsche und spanische Verben (studieren/estudiar; lernen/aprender; pauken/empollar) hinsichtlich ihrer Kookkurrenzen. Die lexikographische Darstellung der Bedeutung der verschiedenen Konstruktionen erscheine häufig nicht im Wörterbuch. Im Korpus können nach Sánchez Hernández Lesarten belegt werden, die in Wörterbüchern nicht vorkommen. So könne z. B. Vokabel als häufigste Kollokation zum Verb pauken nachgewiesen werden. Kontrastive Analysen dienten sowohl in der Ausgangs- als auch in der Zielsprache dem Erkenntnisgewinn. Sie zeigten, bei welchen Verben es Gemeinsamkeiten bzw. Unterschiede in ihrem syntagmatischen Verhalten gebe.

In ihrem Beitrag Verbbedeutung, Konstruktionsbedeutung oder beides? Zur Bedeutung deutscher Doppel-Objekt-Strukturen stellte Kristel Proost das Zusammenspiel von Verbbedeutung und Konstruktionsbedeutung in deutschen DoppelObjekt-Strukturen dar. Nicht für alle Strukturen der Form $\mathrm{NP}_{\text {nom }} \mathrm{V} \mathrm{NP}_{\text {dat }} \mathrm{NP}_{\text {akk }}$ und $\mathrm{NP}_{\text {nom }} \mathrm{V} \mathrm{NP}_{\text {akk }}$ PP mit der Bedeutung , $\mathrm{X}$ bewirkt, dass $\mathrm{Z}$ hin zu einem Ziel/weg von einer Quelle bewegt wird' reichten die Erklärungsansätze des single meaning approach und des multi-meaning approach aus. Proost belegte, dass sich Argumenterweiterungen und Koerzionsphänome nachweisen ließen, die zeigten, dass sich die Gesamtbedeutung einer Konstruktion nicht in allen Fällen durch die Bedeutung des Verbes bestimmen ließe.

Aus der DaF-Perspektive beleuchtete Brigitte Handwerker mit ihrem Beitrag Verfügbarkeit vs. Produktivität von Konstruktionen. - Zum Format von sprechlernrelevanten Informationen im Bereich der Orts- und Zustandsveränderung den Themenbereich der erwerbsoptimierten Beschreibung von Chunks. Dabei gelte es, lerntheoretische Ansätze mit der konstruktionsgrammatischen Erfassung der als Chunks verarbeiteten Einheiten zu verbinden. Aus lerntheoretischer Sicht müsse die Zahl der mögliche Einheiten eines Chunks auf $7+/-2$ begrenzt werden, da dies der optimalen Größe für das Arbeitsgedächtnis entspreche. Nicht der authentische Input stehe im Vordergrund, sondern die Aufmerksamkeitssteuerung durch einen manipulierten Input. Als sprachlernrelevant gelte, neben einem angemessenen Abstraktionsgrad der Konstruktion, auch eine gebrauchsbasierte Einschätzung der Produktivität einer Konstruktion.

Zum Abschluss der Tagung referierte Stefan Engelberg über die Kognitive Organisation von Argumentstrukturen im Lichte quantitativer Studien zu PsychVerben. Die zugrunde liegende Studie untersucht die Verhaltensprofile von 200 Verben mit dem Ziel einer exhaustiven Satzmusterrecherche. Auf der Basis eines annotierten Korpus ließe sich nachweisen, dass viele Argumentstrukturen in unterschiedlicher Häufigkeit auftreten. Engelberg differenziert zwischen der absoluten Häufigkeit einer Argumentstruktur bei einem Verb und der relativen Häufigkeit von Argumenten bei Verben. Dabei gebe es starke Präferenzen für einzelne Muster, d.h. während einzelne Verben selten mit einem bestimmten 
Argumentstrukturmuster auftreten würden, gebe es andere Verben, für die genau diese Argumentstrukturmuster dominierten. Der Zusammenhang zwischen Entrenchment, also der Repräsentationsstärke, und Sprachproduktion könne über den kognitiven Aufwand bzw. die kognitive Leichtigkeit der Sprachproduktion beschrieben werden. Bei hohem Entrenchment könne von kognitiver Leichtigkeit in der Sprachproduktion ausgegangen werden, bei niedrigem Entrenchment von kognitivem Aufwand. Bei den untersuchten Psych-Verben ließen sich Parallelstrukturen mit Partizipien und Adjektiven finden (faszinieren vs. faszinierend; ärgern vs. ärgerlich).

Das Ziel der Tagung war, eine Auseinandersetzung der Valenzgrammatik mit konstruktionsgrammatischen Ansätzen anzuregen. Dabei wurde deutlich, dass sich die Positionen z. T. diametral entgegenstehen. Die Ansätze reichen von einer Einbettung der Valenzgrammatik in die Konstruktionsgrammatik (K. Welke) über eine getrennte Betrachtung von Valenz- und Konstruktionsansätzen in Abhängigkeit von der Betrachtungsperspektive (A. Stefanowitsch) bis zu einer Einbettung der Konstruktionsgrammatik in die Valenzgrammatik (V. Ágel). Für jede Position gibt es eine Reihe von Argumenten. Die Tagung hat gezeigt, dass zwischen Valenz- und Konstruktionsgrammatik eine enge Beziehung besteht, die weiter in den Blickpunkt des Forschungsinteresses gerückt werden kann und soll. 